\title{
A new perceptual activity approach to scientific historiography
}

\author{
Anna Storozhuk \\ Institute of Philosophy and Law of SB RAS, Novosibirsk, Russia
}

Email address:

stor71@mail.ru

\section{To cite this article:}

Anna Storozhuk. A New Perceptual Activity Approach to Scientific Historiography. International Journal of Philosophy.

Vol. 1, No. 1, 2013, pp. 21-28. doi: 10.11648/j.ijp.20130101.13

\begin{abstract}
Beginning from the second half of the XX century, the sociological approaches have been predominant in studying the issues concerning historiography of science; at the same time, the positions of rational philosophy have considerably weakened. The purpose of this article is to develop an alternative approach to the science historiography based on the assumption of perceptual activity. The active approach presupposes a close connection between the rational and the empirical cognitions that allows developing a new method of description of the scientific history. In the foundation of the approach being developed, there is an assumption about a certain perceptual expectation that plays an important role both in perception and rational interpretation of what has been observed. This new view on the relations between scientific theory and experiment allows revealing a larger variety of the occasions of such relations which turn out to be missed under other approaches to the history of science.
\end{abstract}

Keywords: Historiography of Science, Theory-Laden Observations, Theory and Experiment, Activity of Perception

\section{Introduction}

The development of science used to be considered as accumulation of new knowledge. The scientific progress, in turn, was understood as discovering new facts, deriving empirical laws, applying the known theories to new areas, improving the measuring devices and equipment. One of the indications of the development of science was technical progress, which pointed out to the oncoming discovery of truth.

In the 1960-s in the historiography of science there took place a radical turn, initiated by the famous T. Kuhn's book The Structure of Scientific Revolutions (1962). T. Kuhn has expanded the scientific theory concept, having included there the metaphysical assumptions about the world structure. He gave a name of 'paradigm' to this expanded concept, and the work of scientists, according to Kuhn, consisted in reproduction of a given sample. Following a paradigm presupposed a number of irrational elements and required invoking sociological, anthropological and historical approaches. Kuhn has also broadened the understanding of the role of experiment in science, having shown the possibility of a stage of initial data accumulation in the absence of theory, the possibility of using experiment to adjust the paradigm and so on.
This new approach to the description of the science history raised novel questions. Expansion of the concepts of experiment and theory demanded rethinking of their relations. This article is devoted to consideration of the relations between theory and experiment in the context of the science history. The purpose of the article is to strengthen the rational approach in the science history, which is based on the understanding of theory and experiment as more closely connected foundations of scientific activity. In the foundation of the approach being developed here, there is an assumption about the perceptual activity, one of whose manifestations is the perceptual processing of information and its comparing with the expectations. The degree of conformity to the expectations defines a further strategy of the researcher which can be quite diverse. Thinking that one of theory's tasks consists in formulation of the researcher's expectations concerning the reality, we reformulate the relations between theory and experiment in the language of expectations and conformity to them of the perceptual data.

As a result, a unified description of the scientific activity is constructed and a more detailed classification of possible relations between theory and experiment is obtained. Further, we give a description of the science development without engaging social, anthropological and other 
irrational factors. Based on the introduction of the notion of a non-linguistic structure, the mechanism of the scientific search and discovery of new knowledge is revealed, the predictive role of the theory is analyzed in detail, and a constructive function of the theory-ladenness is demonstrated.

\section{Brief Review of the History of Historiography}

The basic philosophy of science is positivism, in whose frameworks the logical positivism was the most developed branch. The traditional positivist approach to studying the relation of theory and facts consisted in the confidence that science has in its disposal a special method based on logic and experiment. With the help of logic, statements are analyzed; by means of experiment they are verified. From the point of view of logical positivism, the development of science is a cumulative process of the knowledge stockpiling. The approach of logical positivists has a number of shortcomings:

\subsection{Some Problems of Historiography in Positivism}

- from the point of view of logic, one of the main methods of obtaining the scientific knowledge, the inductive one, is logically unsound, because a theory can be only falsified by experiment but not confirmed. The facts can only refute a theory (falsification), hence the empirical scientific practice does not correspond to the logical rules;

- the logical approach is not compatible with the historical one and, therefore, is not suitable for the description of the scientific knowledge progress. Some time after, the attempts were made to expand the logical approach by placing consideration into historical context (the methodology of scientific research programs by I. Lakatos (1978)). Its main shortcoming is its being retrospective: a considerable role in determining the status of the scientific theory is played by the decisive contradiction.

- many important cognitive and methodological aspects, being looked at from the logical prospective, turn out to be missing; limitations of the logical framework do not allow answering the questions about the sources of scientific problems and their significance, about the possibility of alternative ways of science development.

- logical approach is vulnerable to a number of critical arguments such as theory ladenness of observations [1] W. V. Quine's thesis of underdetermination of the theory by data [2], L. Fleck's statement about the historical development of scientific fact [3] and other.

The listed problems have demanded an expansion of the scientific theory concept and its inclusion into the historical context.

\subsection{The Historiography of Science Later: Socio-Cultural and Anthropological Approaches}

A well-known book by T. Kuhn The Structure of Scientific Revolutions has raised a question about the relations between history and philosophy of science. Kuhn has pointed out to the non-cumulative character of the development of science: there are many rejected theories, and the experimental data are rejected together with the theory explaining them if they cannot get new interpretation. It was considered before Kuhn that the history of science was a source of the materials, with the help of which philosophers confirmed their concepts or verify them. Kuhn noted that these ideas originated from studying the ready scientific achievements, but such ideas lead astray from the main ways of science: a concept of science drawn from them is no more likely to fit the enterprise that produced them than an image of a national culture drawn from a tourist brochure or a language text [4].

The task of historians was to create a new image of science which would represent more adequately the real activity of scientists. It was important to explain why this, not that theory from the competing ones was selected; why these, not those experiments were conducted first; and why the scientific problems were being solved by these, not those methods. To study the raised questions, there were engaged psychological, sociological, and anthropological techniques. To substantiate the possibility of application of such techniques, the externalist thesis [5] was used, which asserts that insofar as the activity of scientists is a social phenomenon, the political, social or economic factors have a considerable influence on the destiny of both general scientific and particular scientific theories.

The understanding of science as a cultural activity means determination and interpretation of sophisticated collections of actions, signs, beliefs, by means of which the scientists created the sense of their everyday life and work. This approach is applied by anthropologists, who, while studying rather small groups of people, try to reconstruct ancient or geographically remote cultures. The anthropological approach, when applied to small collectives of practicing scientists, looks for understanding of theories and experiments not as idealization or natural phenomena but as a part of the daily life experience of a group of people.

Nevertheless, neither ethnographers nor historians of science are capable of producing absolutely neutral descriptions. Identification and description of cultural characteristics depends on the differences and contradictions of various cultures. Recognition of the properties of other cultures and their analysis are possible because other cultures are not similar to our culture which appears to us as self-evident. Studying cultures turns out to be deformed by the outlook, and the explanation of the anthropologist is not the only one. Because of that, it makes sense to consider the same object from various positions.

In studying modern science there arises the problem of distancing oneself. Due to impossibility of obtaining the time remoteness, the spatial one is applied. The anthropological approach becomes fruitful when it is 
applied to modern but geographically separated centers of scientific activity.

One of the tasks of the anthropological approach is overcoming the precipice between theory and practice: while the experiment is a kind of manual laboratory work and is localized in space and time, the theory is an isolated ready product which overcomes time limitation and crosses over the threshold of the place of its creation, expanding the limits of existence. To fix the imbalance, there are considered particular aspects of various practices of the scientific community. In particular, P. Galison (1987), drawing from one episode of the theoretical physics development, the development of high-energy physics, shows that the experimental research has priority and the theory is considered from the instrumental point of view. Feynman was permanently interested in the space of solutions more than in the equations of motion and was looking for new prospects of the physical theory.

Let us sum up the discussion of the bases of the sociocultural approaches to the science historiography, the first and most known of which is Kuhn's approach describing the development of normal science as following of a paradigm. Besides engaging the sociological, anthropological and historical approaches to studying science, it is important to notice a change of the accents. However, there remain a number of problems which cannot be solved within the framework of the socio-cultural approach:

- the very notion of social factors is still so vague that trying to explain the scientific practice through them means expressing one unknown entity through another, and this is not productive;

- the descriptions offered by historians, cultural studies scholars and anthropologists are not neutral in terms of values, which leads to the next problem;

- the problem of the description objectivity. In the process of scientific activity, scientists are dealing with the real world, investigating it with the help of certain devices in order to minimize the subjective influence and reproducing the experiments using different methods in order to diminish the systematic errors. The representation of scientific practice as socially conditioned and depending on random factors is not adequate in terms of the scientific investigation standards.

However, the development of sociological approaches has brought some novelty into the investigation of the scientific historiography problems and has pointed to one important feature. Till the middle of the XX century, the changes of scientific knowledge were understood predominantly as the changes of theoretical scientific knowledge. A characteristic feature of the historiographic studies of the last two decades is the departure from understanding of the science development as the development of exclusively theoretical knowledge. In the focus of attention of the historians, sociologists and philosophers of science there is now, first of all, the experimental work.

\subsection{Some Problems of the Experimental Science Historiography}

An important problem of theoretical philosophy is the search for explanations of the general understanding of methods and processes in the scientific practice. As applied to the experimental practice, the search for the general laws is complicated by that the experiment occurs here and now and has local significance. The following section is devoted to the development of an approach to the science historiography from the point of view of the philosophy of activity, which will allow solving the problem of locality of the experimental results.

In the author's opinion, the source of the major problems is inadequate understanding of empirical procedures based on the opposition of theory and experiment. The main presuppositions of this approach can be concisely formulated by the following theses. It is considered that:

- perception provides an isomorphism of mental images and the external world, and its results are trustworthy and epistemologically primary;

- truthfulness of perception is guaranteed by the freedom from rational interpretation. The experience, purified from preconceptions, are given a special epistemological significance;

- the Cartesian ontology is the basis of scientific objectivity and scientific method. The existence of a precipice between soul and body resulted in the possibility to objectively describe the material world without any involvement of subjective qualities of the human observer;

- the scientist is likened to a registering instrument, whose activity is reduced to the recording of facts. The development of science is assumed to be cumulative, the scientific knowledge growth means accumulation of facts and their theoretical explanation.

The major problem of the opposition between rational and empirical knowledge is the lack of clear notions about the ways of providing the veracity of scientific knowledge, objectivity of scientific ontology and the resulted historical and cultural relativism. There are no descriptions of the mechanism of setting the problems, emergence of the new problems, and of the processes of scientific search. The indicated problems lead to the necessity of applying new conceptual approach to the description of the activity of scientists.

\section{Development of Science from the Standpoint of the Philosophy of Activity}

Here a new approach to the historiography of science will be developed. First, we will focus on the rational activity of perception: formation of expectations as readiness to perceive this or that sensory information. The role and the task of the considered structure consist in that it forms expectations, manages the search and gathering of 
the necessary information and arranges sensual data. The organizing role of the expectation-forming structure in perception is huge; its presence is a necessary condition of perception. As an example showing the necessity of such preliminary expectation-forming structure, one can use understanding of speech in an unfamiliar foreign language. To the listener, an unknown language seems to be a continuous stream of sounds; while the speech in the native language is recognized even when it is not quite distinct, because the listener has preliminary knowledge forming certain expectations.

Let us give an example showing the importance of preliminary expectations for observation.

\subsection{An Example of the Influence of Preconception on the Possibility of Observation}

Newton adhered to the hypothesis about the corpuscular nature of light; i.e., that light is represented by the linearly spreading particles. Prior to Newton's works, the interference and diffraction phenomena had been investigated and described in detail by Grimaldi, Hooke and Huygens, supporters of the wave theory. Newton had been reproducing many experiments on interference and diffractions of beams but he seemed to 'have ignored or overlooked diffraction effects of the use of a small hole as image, though these had been noticed by his contemporaries' [6]. At the same time, he had possibility to observe the manifestation of the light ray diffraction, when there takes place deviation of the beam from a linear way and increase of the sizes of the shade casted by a small object. However, Newton did not notice the internal strips of light appearing due to diffraction in the object's shade. August Fresnel wrote that it was difficult to understand how the light bending toward the internal part of the shade could have escaped notice by so skilled an observer as Newton. Most likely, it was due to his theoretical biases which closed, to some extent, his eyes on the significant phenomena which could weaken the positions of his principle.

The experiment of Grimaldi on the crested fringes within the shadow, together with several others of his observations equally important, has been left unnoticed by Newton. Those who are attached to the Newtonian theory of light, or to the hypothesis of modern opticians founded on views still less extended, would do well to endeavor to imagine anything like an explanation of these experiments derived from their own doctrines; and if they fail in the attempt, to refrain at least from idle declamation against a system which is founded on the accuracy of its application to all these facts, and to a thousand others of a similar nature [7].

The given example shows the importance of the preliminary conception for the perception process. A change of the physical readiness for perception may create adverse conditions for those irritants which are not in the center of attention. When the readiness to perceive the phenomenon is lacking, it can remain unnoticed; and this is especially so for the hardly noticeable effects.

This structure is of a lower level than language or the perception data, and serves as their general uniting base. The data are always theoretically laden but, nevertheless, they can be used to verify the theory not on the language level but on a lower structural level. The theory establishes a structure, the data fill it. Although the same data can be arranged differently, the data do not have to correspond completely to the proposed structure. Now we consider various types of discrepancy between theory and data.

\begin{tabular}{ccccc}
\hline & Case 1 & Case 2 & Case 3 & Case 4 \\
\hline Expectation & + & + & - & - \\
Data & + & - & + & - \\
\hline
\end{tabular}

Case 4 is trivial and will not be considered. The remaining cases are in the foundation of various ways of treating the relation between theory and experiment. The magnitude of the discrepancy between theory and data can lead to various reactions of scientists; therefore, for each case we should distinguish strong and weak discrepancy.

\section{Case 1}

In the real practice of science, complete conformity of theory and experiments is achieved by no means always. Under the presence of good agreement, the theory is transferred to the level of an instrumental one. As an example, we can indicate the quantum electrodynamics, which is being used for the calculation of the probabilities of the processes in the high energy physics and which provides the calculation precision up to the 11-th digit. However, as a rule, perfect agreement is not achieved.

- Data can correspond to the structure in whole but deviate from the expectations in details, in the attempts of further refinement. In this case a change is possible of both the data and theoretical expectations. One example of the situation, when in case of discrepancy between theoretical expectations and facts not a theory but a well-known fact gets reconsidered, is Fresnel's prediction of the wave theory of light. According to the wave theory of light, in the centre of the opaque disk there should be a light spot. In this case, the prediction of the theory contradicted the wellknown fact: the shade from the solid object should be continuous.

Poisson noticed that the integrals served to calculate the intensity of the diffracted light can be calculated precisely for the center of the shade of a small round opaque screen and for the center of the conic projection of a small round aperture. In the first case, they yield the same intensity as in the absence of the round screen; in the second, they give the intensity varying with the distance and equals zero for several values of the distance; they are governed by a very simple law. Fresnel was suggested to experimentally check these two cases, unforeseen and paradoxical for his theory; and the experiment perfectly confirmed them [8]. This simple example shows invalidity of the position of naïve falsificationism: in the case of contradiction between theory and fact, there can be discarded not the theory but the fact. There are known the cases, when theoretical constructions 
are reconsidered due to incomplete conformity of the theory and the fact. As an example, it is possible to point out to the history of Kepler's studying of the Mars orbit. At first, Kepler assumed that Mars moved along a circular orbit but the deviation of the observation data and the calculations amounted approximately to 8 seconds. In spite of the fact that the usual observation error at that time was 10 minutes, Kepler was not satisfied with the obtained results. The search for more precise conformity of the calculation results and the observation data has led Kepler to the discovery of ellipticity of the planetary orbits [9].

However, incomplete conformity of theory and data very often does not lead to any changes in the theory or discovery of new facts. In such cases, incomplete conformity of theory and data may last for centuries as it was in the case of the displacement of Mercury's perihelion, when the predictions of Newton mechanics differed from the observation data by several dozens of seconds.

- Paradoxes or significant divergences of the expectations and the observation data. It is this case that is traditionally considered in the philosophy of science as the case of discrepancy between theory and facts and is known as falsification. The cases of paradoxes are, indeed, very important as they often play a pilot role in the formation of many fundamental scientific theories which have considerably changed our world outlook by working out new conceptual schemes. In such fashion, the ultraviolet catastrophe served as an initial stimulus for the development of quantum mechanics; Einstein's mental experiment which showed impossibility of a standing electromagnetic wave became a starting point for the creation of special relativity theory; Ehrenfest's paradox [10] about impossibility of fast rotation of an absolutely rigid disk had led to the necessity of usage of nonEuclidean geometry in the general theory of relativity [11].

However, a direct contradiction is not always the factor stimulating the development of the theory. Contradiction plays a destructive role, destroying the existing concepts. The progress of science is more often stimulated by a small divergence between theory and experiment. It is the desire to eliminate the mismatch between expectation and data that compels to search for a compromise by way of both specification of the theory and increase of the accuracy of the experiment.

\section{Case 2}

- Data can leave lacunas, unfilled parts of the structure; and, as an example, we can point out to the discovery in 1870th by the Russian chemist Dmitry Mendeleyev of the Periodic Law at the time when some elements were not known. He was able to predict the existence of the still undiscovered elements; in particular, there were vacant positions below aluminium, boron and silicon. These unknown elements were named by Mendeleyev as 'ecaaluminium' (discovered in 1875 by the French chemist Paul Emile Lecoq de Boisbaudran who named it gallium in honor of his native land) [12], 'eca-boron' (discovered in
1879 by the Swedish chemist Lars Nilson and named scandium) [13], 'eca-silicon' (discovered in 1886 by the German chemist Clemens Winkler, professor of the Freiberg Mining Academy, who named it germanium in honor of his country) [14]. Usually such type of discrepancy stimulates the search of the missing information, that is, the further gathering of data. The scientific theory which offered the structure, which cannot be completely filled by the already known data, possesses a special advantage, the predictive capacity. Such theory is empirically fruitful and leads to discovery of new facts.

- Finally, theoretical expectations may fail, that is, there might be no data available to fill a certain theoretical scheme. For example, the prediction of existence of fractional electric charges or quarks was not justified by the direct experiment [15]. The search of quarks was undertaken by Morpurgo [16], who did not manage to detect fractional electric charges. Impossibility to observe a certain effect oftentimes serves as theoretical answer to the negative result of the experiment. In this case, there was postulated the confinement principle, that is, quarks' nonescaping from hadrons.

\section{Case 3}

- Data can turn out to be superfluous, as for example, 'superfluous' spectral lines in the Bohr's model of atom. In 1912, Niels Bohr proposed a model of the hydrogen atom [17] but the experiments showed that in the strong magnetic field the magnetic lines of atoms split. Such kind of discrepancy as superfluous data stimulates further refinement of the theory. In the given case, Bohr's model was specified by Arnold Sommerfeld who applied the ideas of relativity theory to the calculation of the orbits' shapes and proved that the orbits can be circular or elliptic [18]. The orbits can be oriented in the magnetic field only under certain angles. These additional states allowed explaining the additional spectral lines, and Sommerfeld's model of atom was in good accord with the experimental data [19].

- Unexpected discoveries. Besides the four mentioned kinds of contradictions between theory and facts, there are also the cases of discovery of unexpected facts. As an example, we can mention the discovery of muon [20], which was greeted by the phrase 'who ordered this?' Muon was the first particle for which no place could be found in the existing at that time classification of particles. As a rule, discovery of unexpected data stimulates further development of conceptual means. However, if the new fact does not get theoretical interpretation and, together with it, a possible explanation, then most likely it will not be widely known and later will be silently forgotten.

Let us sum up. From the active approach standpoint, in the basis of both perception and thinking there lie some lower-level structures uniting them. They arrange experiment and can be partially expressed in language. Genetic unity of rational and empirical knowledge, which is the starting point of the philosophy of activity, compels to look differently at the relation of theory and experiment. 
The task of the scientific theory consists not only in creation of some language to describe the ready 'invariable' sensual data, the scientific theory also establishes a certain way of vision, forms the structures which arrange in some manner the sensual experience. These structures are reflected also in the language of the scientific theory, and play an organizing role in perception. The logic reflects the structure and sets the relation and position of its parts, experiment fills the structure with data and specifies the details of the elements involved in the structure. The data are always laden in the sense that the experiment gets ordered and generalized from the very beginning. Besides, as it is shown by the example of verification of Fresnel's wave hypothesis, not the theory but the well-known fact may get discarded.

The active approach reveals those aspects in the theoryexperiment relations that are usually overlooked. Also, the domain of applicability of falsificationism is usually limited to the case of considerable divergence of the theory prediction and the obtained data. As it is shown by the example of the Fresnel's wave hypothesis verification, it is the well-known fact, not the falsified theory that may be discarded.

It is important to note that the facts do not contradict directly to the theory in the sense of logical contradiction, because theory and observation have different nature.

no factual proposition can ever be proved by experiment. Propositions can only be derived from other propositions, they cannot be derived from facts: one cannot prove statements from experiences - 'no more than by thumping the table'. This is one of the basic points of elementary logic, but one which is understood by relatively few people even today [21].

Within the framework of logical approach, contradiction is considered as a relation between two statements, in particular, between a certain statement $A$ of the theory and the statement about the fact "not $A$ ". Statements about facts are not only theoretically laden but also socially and culturally relative. The linguistic tradition, which has gone away from fluctuating matter to the strict world of logic, is by no means fruitless in regard to the question of the theory-fact relations but generates new problems. In particular, an insoluble problem of the linguistic approach is the vicious circle in the argument, arising in the comparison of theory with the theoretically laden facts. However, the scientific theory cannot be exclusively reduced to logic and is not a purely linguistic enterprise; therefore, the discrepancies of theory and facts are more diverse than just logical contradictions.

Let us specify some philosophically significant consequences of the new approach application. Among them, there are a possibility to offer an alternative explanation to the scientific progress, a possibility to consider the heuristic role of experiment in the case of probabilistic laws and a new view on the soul-body dualism.

1) Falsificationism recognizes the change of theories as the progress of science and considers contradiction to be a factor of scientific progress. From our point of view, the major factor of scientific progress is the constant tension which exists between the structure-forming expectations and the structure-filling data. The significance of this result consists in a possibility to explain scientific progress from rational positions as aspiration to conformity between theory and data, which quite agrees with the understanding of empirical adequacy introduced in Van Fraassen's constructive empiricism [22]. Thereby, a refutation is offered to the No-Miracle argument which claims that aspiration to the truth is a unique explanation of scientific progress.

Progress does not necessarily mean aspiration to the truth. For example, biological evolution has no purpose at all. We may argue about the reason of technical evolution economic interests or aspiration to comfort - but, in any case, it is not the search for the truth. The reasoning in the language of mental structures, which form expectations and prepare the observer to the perception of information of a certain kind, allows explaining both empirical and theoretical progress as bringing to conformity expectations and data. The search for conformity, not aspiration to the truth is a major factor of scientific progress; and the development of new theories can be considered as the search for new structures which order the data differently.

2) The traditional point of view that data verify theory is inapplicable in the case of probabilistic laws. If there is 98 percent probability to get a white ball out of the urn and you get a black one 100 times in a row, this fact is by no means a refutation of high probability of getting a white ball. As it is well known, even at the 98 percent probability for a white ball, the probability theory admits the possibility (although a very unlikely one) of getting a black ball $10,100,1000$ or an arbitrary finite number of times in a row. The falsificationism approach is inapplicable in such cases, because the probabilistic law is not falsifiable: it cannot be absolutely refuted by any finite number of trials. Let us note, first, that the predictions of the theory may have probabilistic character, and, second, the relations between theory and facts are far from being restricted to the theory verification. The data may point out to unrealized expectations and thus stimulate further scientific search, even without entering at all into direct contradiction with the theory.

3) The problem of the soul-body dualism was set by Descartes who, following his separation of the substances on the thinking one and the extended one, postulated independence of soul and body. After that, there arose a question about the possibility of explanation of the psychophysical unity, allowing explaining the coordinated mental and physical actions. This issue was widely discussed in Modern philosophy. The assumption of the existence of the structures, common both for language and perception, enables us to look differently at the problem of the theory-tofacts transition. Since the structure unites both body (for it is physiologically a part of nervous system) and mind, it can play the role of a bridge thrown over the precipice between mind and body. 


\section{Conclusion}

The issue of how to write the history of science became topical after T. Kuhn's indication of the non-cumulative character of scientific knowledge. His work opened the way for the sociological approach, which, however, could not overcome the vagueness of the notion of a social factor. At the same time, the positions of rational philosophy, which accepts rigorous distinction between theory and observation, were weakened by criticism. The problems in the foundations of empiricism caused a wave of interest towards the experimental work; however, many philosophers are trying to be saved from theory ladenness of observations by denying it.

We do accept theory ladenness; and the goal of the article is to construct an approach to the science historiography based on the absence of strict division between theory and experience. The proposed typology of the relations between theory and experiment is grounded on new conceptual foundations. Their revision is caused by the necessity of overcoming a number of skeptical arguments, threatening the foundations of empiricism. Theory ladenness is one of the obstacles, which hinder the generation of empirical judgements, comparison of theory with experience and which devaluate the epistemological significance of experience.

The hypothesis about the existence of some lower-level structures, which lie at the foundation of both rational activity and perception, allows providing genetic and structural affinity of the cognitive processes. Structure is a more general notion, including both perceived information and theoretical concepts. It allows considering them while avoiding their contraposition.

The heuristic value of the approach, suggested in the article, is in the possibility of equal analysis of the cognitive roles of theory and experiments, which allows introducing a typology of their relations. The relation between theory and data is considered from the point of view of conformity of experiment to the theoretically generated expectations. This allows considering a larger number of cases than it is possible from the standpoint of traditional approaches where experiment is supposed to be passive reflection of the reality. In particular, the data can leave lacunas, blank parts of the structure, or, on the contrary, the data may turn out to be superfluous. The data may correspond to the structure as a whole but deviate from the expectations in some details in the attempts of further refinement. The unexpected discoveries are possible or, on the contrary, the theoretical expectations may not be realized, that is, there may not be enough data to fill a certain theoretical scheme. Finally, considerable deviations between the expectations and the observation data are possible. It is the very case that is traditionally considered in the philosophy of science as the case of theory's unconformity to the facts and is treated as a case of falsification of the theory.

Our analysis demonstrates more elaborate classification of theories and experiments than the logical approach, which is restricted to distinguishing two cases: falsification and verification. From the active approach viewpoint, the task of scientific theory consists not only in creation of some way of description and explanation of the ready 'invariable' sensual data but also in the task of setting a certain way of vision and forming the structures which arrange the sensual experience in one way or another. These structures are reflected in the language of the scientific theory and play an organizing role in perception, directing the search. Logic reflects the structure and sets the relation and position of its parts; experiment fills the structure with data and specifies the details of the elements entering into the structure. The data are always laden in the sense that experiment is arranged and generalized from the very beginning.

The approach being developed possesses the forecasting function and allows predicting the main direction of the scientific activity, provided the discrepancy with the theoretical expectations can be identified. The most fruitful for the development of science is a small deviation between the data and the theoretical expectations. The search for conformity is a major factor of scientific progress; and the development of new theories may be considered as the search for new structures, which arrange and order the data differently.

The advantage of the suggested position is in reevaluation of the role of theory ladenness and its turning from being a critical argument to being a certain feature of cognition which possesses an important heuristic function. In the area of philosophy of scientific experiment, where the construction of classifications of the means and ways of research is difficult, the presented approach is a version of heuristics allowing describing various cases of the theoryexperiment relations.

\section{References}

[1] P. Duhem "The Aim and Structure of Physical Theory". (P. P Wiener, Trans. from $2^{\text {nd }} 1914$ ) originally published as La Théorie Physique: Son Objet et sa Structure (Paris: Marcel Riviera \& Cie.). Princeton, New Jersey: Princeton University Press, 1954. (First published 1906)

[2] W.V. Quine, "Two Dogmas of Empiricism", reprinted in From a Logical Point of View, 2nd edition, (pp. 20-46.) Cambridge: Harvard University Press. (First published W. V. Quine, (1951) "Two Dogmas of Empiricism". The Philosophical Review, 1953/1980, v. 60, 20-43.)

[3] L. Fleck, "The Genesis and Development of a Scientific Fact", (edited by T.J. Trenn and R.K. Merton, foreword by Thomas Kuhn). Chicago: University of Chicago Press, 1979.

[4] T. Kuhn, "Structure of Scientific Revolutions". Chicago: University of Chicago Press, 1962/1970.

[5] D. Bloor, "Knowledge and social imagery". Chicago: University of Chicago Press, 1991; B. Barnes "Scientific Knowledge and Sociological Theory". London; Boston: Routledge and K. Paul, 1974. 
[6] R. Harré, "Great scientific experiments: Twenty experiments that changed our view of the world". Oxford: Phaidon Press Ltd, 1981.

[7] A. Fresnel, "Memoir On The Diffraction Of Light" In H. Crew (Ed.), The Wave Theory Of Light Memoirs By Huygens, Young And Fresnel. (pp. 79-144). New York, Cincinnati: Chicago American Book Company Crew, Henry, 1859, p.73-74.

[8] E. Werde, "The Works by Augustin-Jean Fresnel". In Rodichev V. I. (Ed.) Creators of Physical Optics, 1973. (pp. 180-206, pp. 194-195).

[9] A. Ede, L. B. Cormack, "A History Of Science In Society: From Philosophy To Utility". Toronto: University of Toronto Press, 2004, p.130.

[10] O. Gron, S. Hervik, "Einstein's general theory of relativity: with modern applications in cosmology". New York: Springer, 2007, pp.90-91.

[11] S. Weinberg, "Gravitation and Cosmology: Principles and Applications of the General Theory of Relativity", 1972, p.91

[12] R. Duncan, "Elements of Faith: Hydrogen to Tin, Faith Facts and Learning, Lessons from the Periodic Table". New Leaf Publishing Group., 2008, p.66.

[13] L. Rezende, "Chronology of science". Infobase Publishing: Prints India, 2006, p.222.
[14] M. D. Gordin, “A well-ordered thing: Dmitrii Mendeleev and the shadow of the periodic table". Basic Books., 2004, p. 42.

[15] H. G. Dosch, "Beyond the nanoworld: quarks, leptons, and gauge bosons". A K Peters, Ltd, 2008.

[16] A. Pickering, "The mangle of practice: time, agency, and science". Chicago: University of Chicago Press, 1995, p.71.

[17] K. A. Peacock, "The quantum revolution: a historical perspective". Greenwood Publishing Group, 2008, p.36.

[18] H. Haken, H. Wolf, "The Physics of Atoms and Quanta: Introduction to Experiments and Theory". Springer-Verlag Bertia Heidelberg, 2004.

[19] D. M. Greenberger, K. Hentschel, F. Weinert „Compendium of Quantum Physics: Concepts, Experiments, History and Philosophy“. Berlin, Heidelberg: Springer-Verlag, 2009, p.60.

[20] K. Nagamine, "Introductory muon science". Cambridge: Cambridge University Press, 2003.

[21] I. Lacatos, "The methodology of scientific research programmes: Philosophical Papers" Volume 1 Cambridge: Cambridge University Press, 1978/1980, p.16.

[22] Bas. C. van Fraassen, "The Scientific Image". Oxford University Press, 1980. 